\title{
Terapia fotodinámica en el cáncer de próstata localizado
}

\author{
Ramírez Backhaus M, Trassierra Villa M, Vera Donoso CD, Jiménez Cruz JF. \\ Servicio de Urología. Hospital Universitario La Fe. Valencia.
}

Actas Urol Esp. 2007;31(6):633-641

\section{RESUMEN}

\section{TERAPIA FOTODINÁMICA EN EL CÁNCER DE PRÓSTATA LOCALIZADO}

La terapia fotodinámica se basa en la aplicación de una fuente de energía en forma de luz con una determinada longitud de onda, sobre un tejido previamente fotosensibilizado mediante un compuesto químico, en presencia de oxígeno; de modo que se generan gran cantidad de radicales libres y derivados del oxígeno (compuestos hidroxilo) que producen necrosis del tejido tratado. El perfeccionamiento de esta técnica en los últimos años ha permitido su reciente desarrollo como modalidad terapéutica del cáncer de próstata localizado. En este momento, se están llevando a cabo varios ensayos clínicos en pacientes con cáncer de próstata órgano-confinados tanto como tratamiento de primera línea como de rescate. No hay riesgo de diseminar el cáncer en los tejidos adyacentes, o de fármaco-toxicidad acumulativa. Por lo tanto, se puede repetir la terapia las veces que se crea necesaria y se puede aplicar sobre un tejido previamente irradiado. De la literatura se desprende que la terapia fotodinámica, en un futuro muy próximo, será una opción terapéutica para los pacientes con cáncer de próstata.

Palabras clave: Terapia fotodinámica. Cáncer de próstata localizado.

\section{ABSTRACT \\ PHOTODYNAMIC THERAPY IN LOCALISED PROSTATE CANCER}

Photodynamic therapy is based on the administration of an energy source in form of light of a specific wavelength, on a previously photosensitized tissue by a chemical compound, in the presence of oxygen, so that the great deal of free radicals and oxygen derivatives generated (hydroxyl compounds) produces necrosis of the treated tissue. Technique improvement during the last years has allowed its recent development as a therapeutic method for localised prostate cancer. At present, several clinical trials are ongoing in patients with organ-confined prostate cancer both as a first line and salvage treatment. There is no risk either of cancer dissemination in surrounding tissues or accumulative pharmaco-toxicity. Therefore, the technique can be repeated as often as needed and can be administered on a previously irradiated tissue. The literature review shows that photodynamic treatment will become a therapeutic option for patients with prostate cancer in the very near future.

Keywords: Photodynamic therapy. Localised prostate cancer.

\section{REVISIÓN HISTÓRICA}

El fenómeno de la fototoxicidad es conocido desde la antigüedad. En tiempos de los egipcios se usaba una combinación de sol y sustancias naturales para tratar diversas afecciones cutáneas. El concepto científico de terapia fotodinámica se conoce desde comienzo del siglo XX cuando Raab comprobó que determinadas fuentes de energia eran letales para los paramecios expuestos a acridina ${ }^{1}$. Posteriormente, Tappeiner utilizó de forma tópica estos tintes para el tratamiento de lesiones dermatológicas ${ }^{2,3}$. En 1913, MeyerBet $z^{4}$ investigó la acumulación de determinados fármacos en tejidos tumorales de ratas, y el aumento de la susceptibilidad de éstos a la irradiación. Figge et al. ${ }^{5}$ investigaron la fluorescencia 
de dichos compuestos y su utilidad para delimitar el margen tumoral. Más tarde, con los estudios de Lipson y Blades se fueron purificando, cada vez más, los compuestos fotosensibilizantes.

Los compuestos químicos fotosensibilizantes más desarrollados en la actualidad son la porfirina y sus derivados ${ }^{5-7}$. Hasta este momento, miles de pacientes han sido tratados con terapia fotodinámica para distintas neoplasias y los primeros ensayos clínicos aleatorizados se iniciaron en 1987. De hecho, la terapia fotodinámica está autorizada en 10 países y la utilizan distintas especialidades. En el Reino Unido está aprobado su uso para algunos casos de degeneración macular asociada a la edad. En otros países también se aplica en patologías premalignas como esófago de Barret, displasias del cuello de cérvix o queratosis actínica. La aplicación como terapia tumoral se inició con las lesiones cutáneas superficiales y prosiguió, gracias a la mejora de los sistemas de irradiación, con el tratamiento de tumores organoconfinados sólidos en cabeza, cuello y páncreas.

El perfeccionamiento de esta técnica en los últimos años ha permitido su reciente desarrollo como modalidad terapéutica del cáncer de próstata localizado.

\section{INTRODUCCIÓN}

La terapia fotodinámica se basa en la aplicación de una fuente de energía en forma de luz con una determinada longitud de onda, sobre un tejido previamente fotosensibilizado mediante un compuesto químico, en presencia de oxígeno; de modo que se generan gran cantidad de radicales libres y derivados del oxígeno (compuestos hidroxilo) que producen necrosis del tejido. (Fig. 1).

Es una técnica doblemente selectiva:

- Se consigue una alta concentración del fármaco fotosensibilizante en el teji- do diana (tumoral), que además, se puede potenciar utilizando diferentes moléculas para aumentar la afinidad del compuesto por determinados tejidos ${ }^{8,9}$.

- Se circunscribe la radiación sobre un volumen concreto de tejido.

- La longitud de onda necesaria para activar el fármaco fotosensibilizador suele estar entre los 600 y los 800 nanómetros (nm). Esto se debe a que lo hemoglobina absorbe casi toda la luz por debajo de los $600 \mathrm{~nm}$ y las longitudes de onda mayores de $900 \mathrm{~nm}$ son insuficientes para generar radicales libres ${ }^{10}$.

- Otra ventaja es que la técnica es reproducible y se puede aplicar de forma repetida sobre el mismo tejido.

- La profundidad que alcanza la irradiación depende de la longitud de onda, del flujo administrado, capacidad de absorción del tejido y de las características intrínsecas del fármaco utilizado (espectro fotométrico, coeficiente de extinción, vida media...) con una compleja relación matemática entre ellas que no procede analizar en el presente capítulo. En líneas generales, se alcanza una profundidad de unos 2 ó $3 \mathrm{~mm}$. con $630 \mathrm{~nm}$ de energía y utilizando 700 u $800 \mathrm{~nm}$ conseguimos 5-6 $\mathrm{mm}^{11}$.

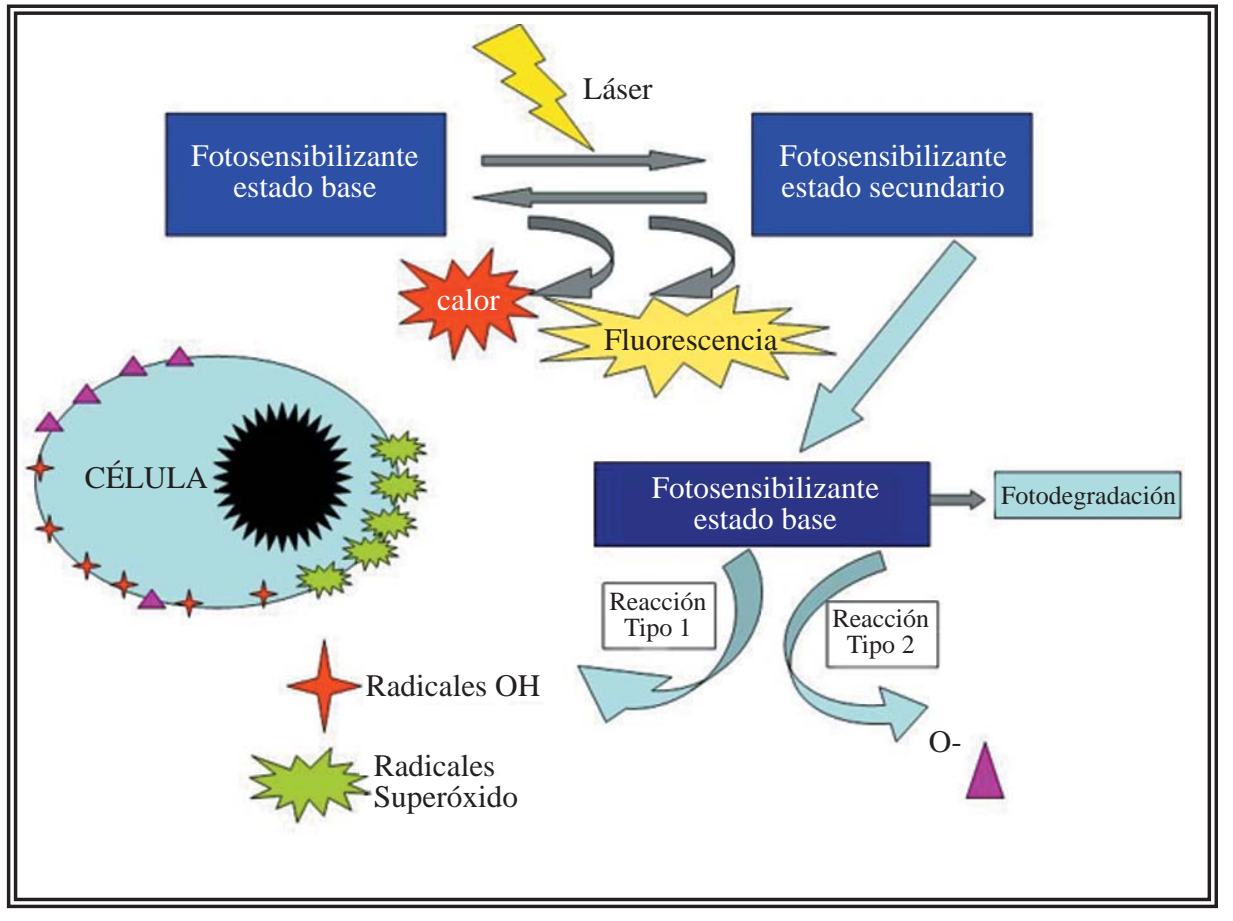

FIGURA 1: Intravascularmente la fuente de energía activa el fármaco en dos fases formando radicales hidroxilo y super-óxido que necrosan la célula tumoral. 


\section{FARMACOS FOTOSENSIBILIZANTES}

-Actualmente, hay muchos fármacos en desarrollo ${ }^{14-17}$. No es nuestra pretensión detallarlos todos, simplemente nombraremos aquellos que se encuentran en un estado avanzado de investigación clínica.

1. Los aniónicos lipofilicos son los fotosensibilizantes por excelencia: Este grupo incluye las hematoporfirinas y sus derivados. La alta fototoxicidad cutánea que poseen (perdura hasta 4-6 semanas) y la escasa activación a las longitudes de onda terapéuticas (600-900 nm) han hecho que caigan en desuso dando paso a formas mas purificadas de la misma familia ${ }^{16,17}$. Por ejemplo los derivados tetrapirrólicos como los benzopirenos, también dentro de esta familia; con mejor perfil farmacocinético, mayor afinidad por el tejido tumoral, menor fototoxicidad cutánea y mejores características fotoquímicas puesto que funcionan bien a altas longitudes de onda ${ }^{18-21}$. Un fármaco en auge para el tratamiento del órgano que nos concierne es el presente capítulo es el TOOKAD (Paladium bacteriumchlorophile) también insoluble en agua y lipofílico. Estos compuestos alcanzan máxima concentración en las células endoteliales de los neovasos tumorales y, por tanto, con la irradiación esta neovasculatura tumoral se necrosa y cesa el aporte nutricional al tejido tumoral. Son, por ejemplo, los agentes de elección para el tratamiento de la degeneración macular asociada a la edad ${ }^{18,22}$.

2. Compuestos catiónicos. Estas sustancias se concentran en las membranas mitocondriales de las células tumorales, debido a que el gradiente eléctrico en estas células es mayor que en las normales ${ }^{23}$. Por tanto y a diferencia de los anteriores, los compuestos catiónicos necrosan directamente la propia célula tumoral. Además, estos compuestos se pueden combinar con los del primer grupo, de modo, que podemos conseguir un doble efecto terapéutico, tanto sobre los neovasos, como sobre las propias células malignas ${ }^{24}$. Hay varios trabajos en la literatura que relatan las experiencias con estos compuestos ${ }^{25,26}$.

3. Recientemente, se ha investigado la posibilidad de administrar un precursor químico sinté- tico inactivo y que la forma activa del fármaco sea sintetizado in situ en las células tumorales. Este sería el caso del aminoácido Alanina (Ala) que participa habitualmente en la cascada de formación del grupo HEM. El último paso en la cascada de formación implica el paso de Protoporfirina IX (con actividad fotosensibilizante) en el grupo HEMO; dicho paso está controlado mediante "feed back" negativo por la enzima ALA Sintetasa. Cuando añadimos de forma exógena el aminoácido, se satura el mecanismo de control y se acumula protoporfirina IX en exceso en las células tumorales; el acúmulo de la sustancia fotosensibilizante da posibilidades no solo terapéuticas sino también diagnósticas ${ }^{27-32}$.

\section{BIODISPONIBILIDAD}

La razón por la que el fármaco se distribuye en mayor medida en el tejido tumoral se debe a varios motivos:

- Mayor índice de proliferación celular,

- Peor drenaje linfático,

- Mayor permeabilidad de los vasos tumorales

- Probablemente también jueguen un papel importante otras interacciones entre el fotosensibilizante y marcadores de las células tumorales así como el incremento de la síntesis de factores de crecimiento como VEGF (Vascular Endotelial Grown Factor) ${ }^{33-36 .}$

- Se sugiere que, a nivel molecular, los responsables de la mayor distribución del fármaco en los tejidos tumorales son las lipoproteinas de baja densidad (LDL), de hecho se detecta una sobreexpresión de receptores de LDL en células malignas y en células endoteliales de los tejidos tumorales $^{37,38}$.

\section{MECANISMOS DE ACCIÓN}

La mayoría de los fármacos citotóxicos actúan a tres niveles; celular, vascular y quizás también inmunológico ${ }^{13,48-55}$.

La localización intracelular del fotosensibilizador es fundamental para la eficacia del tratamiento. A su vez, la distribución intracelular del compuesto dependerá de las características fisicoquímicas del mismo y estado celular de base.

Algunos estudios han demostrado que, los fármacos que por sus características se localizan en las mitocondrias inducen rápidamente a la apop- 
tosis, estableciendo un contraste con los que se concentran en los lisosomas o en las membranas plasmáticas $^{39-41}$.

Los mecanismos de muerte celular probablemente sean lesiones en las organelas celulares como: Peroxidación de las membranas lipídicas, rotura de las membranas lisosomales, pérdida en el potencial mitocondrial transmebrana ${ }^{42}$ y daño a los componentes nucleares ${ }^{43}$. De todos modos, en la mayoría de los ensayos, el daño vascular supone el principal mecanismo de la muerte celular in vivo y se piensa que empieza con la alteración de algunos eicosanoides como los tromboxanos ${ }^{44}$, histaminas o el factor de necrosis tumoral alfa ${ }^{45}$.

Microscópicamente, el daño vascular consiste en eritema agudo, edema y necrosis; microscópicamente, el tejido tumoral sufre lesión de las células endoteliales, agregación plaquetaria, vasoconstricción y hemorragia ${ }^{46,47}$.

En cuanto al papel del sistema inmune en la destrucción del tumor mediado por terapia fotodinámica, Nseyo et al. ${ }^{51,52}$ objetivaron altas concentraciones de (IL)-1Beta, IL-2 y TNF-alfa, en la orina de pacientes tratados con fototerapia para el cáncer de vejiga. El papel de estas citoquinas está por explicar. Otros ensayos han sugerido el aumento de la actividad de células Natural Killers o de Linfocitos T citotóxicos o supresores o, también, la mayor concentración de macrófagos activados tras la aplicación de fototerapia en los tejidos tumorales ${ }^{48,54,56,57}$

En principio, el efecto de esta modalidad terapéutica se consigue cuando se alcanza una concentración de fármaco fototóxico y de cantidad de energía luminosa de forma simultánea. Además de todo esto, es necesaria la presencia de oxígeno ${ }^{58,59}$. Los iones hidroxilo y los aniones superóxido juegan un papel igualmente importante ${ }^{60}$. La $\mathrm{pO}_{2}$ necesaria para la muerte celular va a variar en función del tipo de fármaco y habrá fármacos más y menos dependientes del oxígeno. Los neovasos tumorales no irrigan de manera homogénea al tumor y habrá regiones tumorales más hipóxicas que otras; siendo éste, un factor limitante para la efectividad de la terapia fotodinámica. La incapacidad de monitorizar la $\mathrm{pO}_{2}$ en el tejido irradiado quizás suponga otra traba para optimizar el rendimiento del tratamiento.
Otro condicionante del éxito de la terapia fotodinámica es la selectividad del fármaco por el tejido tumoral. No basta con una mayor concentración de fármaco en el tejido diana; $\mathrm{El}$ objetivo es que el fármaco sea selectivo para el tejido en cuestión y una parte de los esfuerzos de la investigación farmacológica, busca este propósito.

Las dos ramas en desarrollo son:

- Blanco inmunológico "Inmunotargetting". Los tumores generan antígenos nuevos y específicos. Si obtenemos y añadimos al fármaco anticuerpos que reconozcan dichos antígenos tendríamos una selectividad máxima del fármaco por el órgano diana. Hay descritos en la literatura varios estudios acerca de la destrucción celular selectiva con inmunoconjugados para leucemia de células $\mathrm{T}^{61}$ y para tumores vesicales ${ }^{62,64}$, algunos experimentos in vivo para cáncer de ovario en ratones y en melanoma ${ }^{65}$. Los resultados in vitro son prometedores, pero se necesitarán conjugados mas perfeccionados y un estudio más detallado de la farmacocinética de estos compuestos in vivo.

- Dada la importancia de las lipoproteínas de baja densidad en los tejidos tumorales surgió la idea de utilizar complejos fármaco fotosensibilizador con LDL para aumentar la selectividad del fármaco ${ }^{66}$.

\section{APLICACIONES EN CÁNCER DE PRÓSTATA ORGANOCONFINADO}

El desarrollo de la terapia fotodinámica en el cáncer de próstata ha pasado por lógicas etapas: Desde los ensayos sobre ratas a las que se les trasplantaron células prostáticas neoplásicas ${ }^{67,68}$ hasta los ensayos clínicos aleatorizados multicéntricos que están en marcha en la actualidad, pasando por la experimentación en modelos cani$\operatorname{nos}^{69-73}$. Chen et al. utilizando el fotosensibilizante WST 09 en perros, comprobaron que se producían lesiones necróticas de $3 \mathrm{~cm}$ de diámetro con una sola fibra de láser. Posteriormente, se aplicó sobre próstatas tratadas con radioterapia y demostraron que no había diferencias en el tamaño de la lesión ni en los efectos secundarios ${ }^{74,75}$.

La primera constancia del uso clínico de terapia fotodinámica para el cáncer de próstata en humanos data de 1990. Windhal ${ }^{76}$, utilizando derivados de hematoporfirina trató pacientes con 
cáncer de próstata incidental tras una RTU de próstata; un paciente de dicha serie murió por cáncer de pulmón a los seis meses y en la necropsia post morten no encontraron restos de cáncer de próstata. De todos modos, no queda constancia del seguimiento del resto de la serie en la literatura.

El primer ensayo clínico formal para cáncer de próstata en humanos se llevó a cabo en el Servicio de Urología del University College of London en el año $2002^{77}$ donde 14 pacientes con recidiva de cáncer de próstata tras radioterapia fueron tratados con fototerapia. En 9 de los 14 pacientes hubo una reducción importante de los niveles de PSA y se objetivaron signos de necrosis en las ecografias transrectales y biopsias prostáticas de seguimiento.

Los distintos grupos que han utilizado esta modalidad terapéutica se detallan en la Tabla 1 tomada de Moore et al. ${ }^{78}$.

Inicialmente, el paciente tenía que acudir de manera ambulatoria al hospital para la perfusión del fármaco fotosensibilizante y en un segundo tiempo, días después, se realizaba la implantación de las agujas por vía transperineal, por supuesto sin imágenes a tiempo real, para la irradiación prostática. Actualmente, con el desarrollo de los fármacos, en un mismo acto se perfunde el fármaco y se irradia la próstata previa la colocación de las agujas con imágenes a tiempo real. Hoy en día, incluso, se monitoriza el flujo de luz en las distintas áreas de la próstata, uretra y recto, durante el proceso, permitiendo ajustar la máxima intensidad al tejido prostático afecto e irradiar al mínimo los tejidos adyacentes con el fin de reducir los efectos secundarios.

La terapia fotodinámica para el cáncer ha de ser selectiva, es decir no debe afectar a los tejidos adyacentes a la próstata. Además en un modelo con perros, se comprobó que la terapia fotodinámica dejaba indemne el colágeno a las dosis en que necrosaba al tejido prostático ${ }^{73}$. Esto induce a pensar que con las dosis adecuadas de energía y fármaco se puede conseguir destruir el tejido prostático, sin alterar la anatomía perineal, incluso conservando las bandeletas vasculonerviosas $^{79}$ (Fig. 2).

\section{Utilización de blancos vasculares}

Actualmente se está estudiando la terapia del blanco vascular con el fármaco fotosensibilizante palladium bacteriophephorbide TOOKAD ${ }^{\circledR}$. El fármaco se activa en el torrente vascular y daña la estructura vascular del tumor con la consecuente necrosis tumoral. En este momento se están llevando a cabo varios ensayos clínicos en pacientes con cáncer de próstata organoconfinados, tanto como tratamiento de primera línea como de rescate.

Los efectos secundarios de la terapia fotodinámica prostática dependen del fármaco y de la irradiación. Los fármacos más antiguos se acumulaban a altas concentraciones en la piel, lo que conllevaba una fototoxicidad cutánea muy importante durante periodos prolongados. Esto obligaba a los pacientes a evitar la exposición solar por lapsos de tiempo excesivos. Los fármacos más modernos apenas se acumulan en la piel disminuyendo, por tanto, dicho inconveniente.

Para la irradiación es necesaria la punción perineal de los lóbulos prostáticos y los efectos secundarios de la colocación de dichas agujas (prostatitis, hematuria...) se superponen a los de las técnicas como braquiterapia o crioterapia. Se han descrito dos casos de pacientes que presentaron incontinencia urinaria, probablemente a

Tabla 1. Experiencia clínica con la terapia foto dinámica para el cáncer de próstata organoconfinado:

\begin{tabular}{lllll}
\hline Investigador & Año & Publicación & Pacientes & Fármaco \\
\hline Windahl et al. $^{76}$ & 1990 & Carta & Tras RTU & Hematoporfirinas \\
Nathan et al. ${ }^{77}$ & 2002 & Original & Recidiva tras radioterapia & mTHPC \\
Moore et al. & 2003 & Comunicación oral & Tumor primario & mTHPC \\
Hahn et al. & 2003 & Comunicación oral & Recidiva tras radioterapia & Motexafino \\
Zaak et al. & 2003 & Comunicación oral & Tumor primario & Ácido aminolevulénico \\
Trachtenberg et al. & 2004 & Comunicación oral & Recidiva tras radioterapia & TOOKAD \\
\hline
\end{tabular}




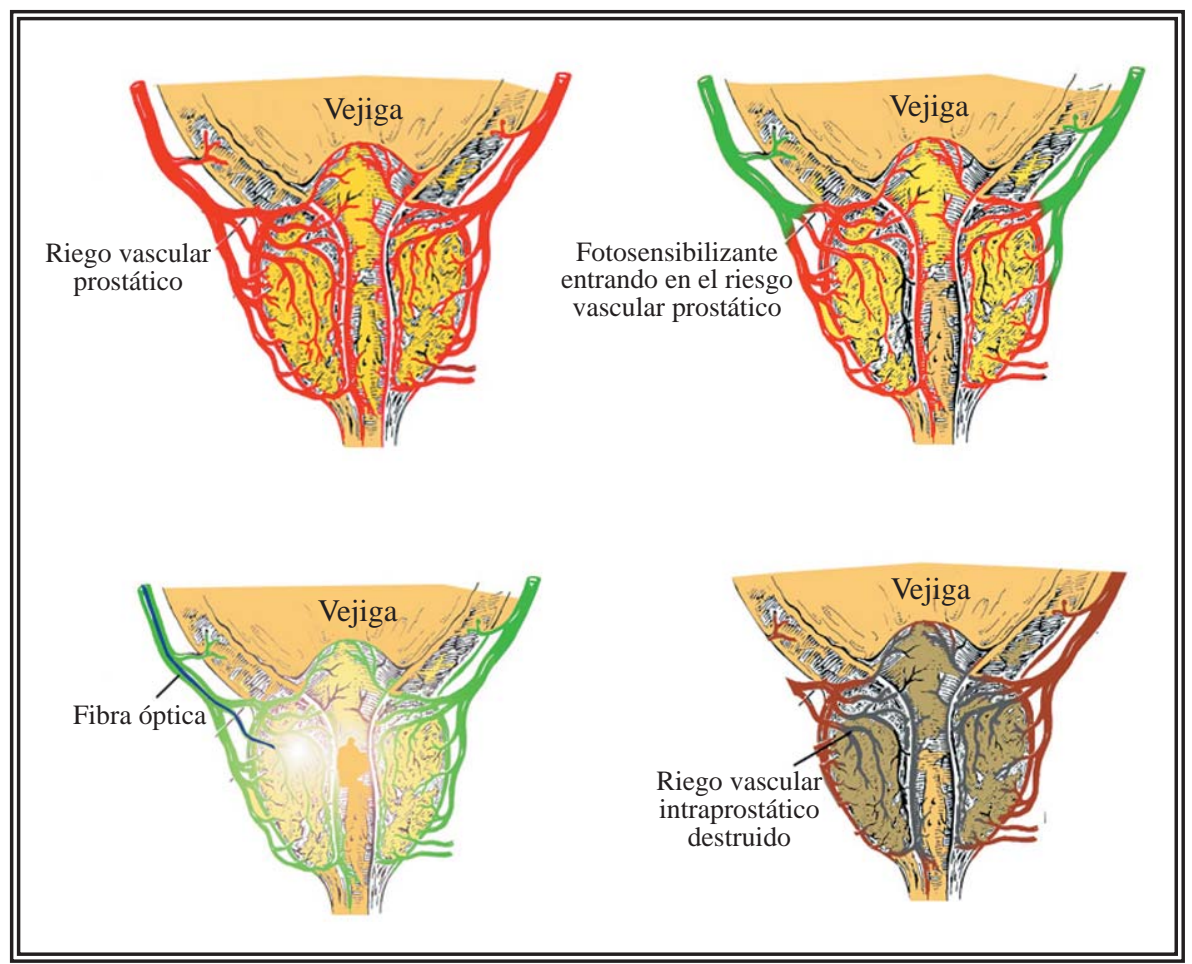

FIGURA 2: Terapia foto dinámica en el cáncer de próstata órgano-confinado. El efecto citotóxico de esta modalidad terapéutica se consigue cuando una fuente de energía láser incide, mediante fibras ópticas guiadas por punción perineal, sobre la próstata fotosensibilizada por la inyección-perfusión de un compuesto químico.

del fármaco y su efecto en el órgano. Actualmente, los nuevos fármacos tiene un período ventana de minutos $\mathrm{y}$ en el mismo acto se perfunde el fotosensibilizante $y$ se aplica la fuente de energía, el efecto curativo sobre los tejidos se objetiva muy rápidamente. No hay riesgo de diseminar el cáncer en los tejidos adyacentes, o de farmacotoxicidad acumulativa. Por lo tanto, se puede repetir la terapia las veces que se crea necesaria y se puede aplicar sobre un tejido previamente irradiado ${ }^{74}$.

Cualquiera de las modalidades terapéuticas ha de ser eficaz y tener una baja morbilidad asociada ${ }^{80}$.

De la literatura se desprende que la terapia fotodinámica cumplirá en un futuro muy próximo los tres requisitos ${ }^{78}$

consecuencia de la necrosis del esfinter externo y otros cuatro casos de disfunción eréctil post tratamiento ${ }^{77}$.

El futuro es prometedor, si bien se observan varios inconvenientes: El primero de ellos reside en las características histológicas intrínsecas de la glándula que redunda en unas propiedades ópticas muy variables. El segundo, es que por el momento no se puede monitorizar de una manera adecuada la concentración intraprostática de la droga, del oxígeno y la intensidad de luz. La interacción de estos factores es muy compleja y por tanto el avance en este punto determinará en gran medida el éxito y aceptación de esta modalidad terapéutica ${ }^{78}$.

\section{CONCLUSIONES}

Mientras no exista un procedimiento que asegure el control absoluto del cáncer órgano-confinado, el paciente elegirá una u otra opción basándose en la menor morbilidad.

Atrás quedan los antiguos fármacos fotosensibilizantes con un largo periodo entre la inyección y será una opción terapeutica para los pacientes con cáncer de próstata.

\section{REFERENCIAS}

1. Raab C. Über die Wirkung fluoreszierender Stoffe auf Infusoria Z Biol. 1900;39:524-526.

2. Von Tappeiner HA, Jensionek A. Therapeutische Versuche mit fluorescierenden Stoffen. Münch Med Wochenschr. 1903; 47:2042-2044.

3. Von Tappeiner HA, Jodlbauer A. Die sensibilisierende Wirkung fluorescierender Substanzen: gesammelte Untersuchungen über die photodynamische Erscheinung. Leipzig, Germany. Vogel. 1907.

4. Meyer-Betz F. Untersuchungen über die biologische (photodynamische) Wirkung des Hämatoporphyrins und anderer Derivate des Blut- und Gallenfarbstoffs Dtsch Arch Klin Med. 1913;112:476-503.

5. Figge FJ, Wieland GS, Manganielleo LOJ. Cancer detection and therapy: affinity of neoplastic, embryonic, and traumatized tissues for porphyrins and metalloporphyrins. Proc Soc Exp Biol Med. 1948;68:640.

6. Kessel D. Tumor-localizing components of the porphyrin preparation hematoporphyrin derivative. Cancer Res. 1983 May;43(5): 1994-1999.

7. Dougherty TJ, Potter WR, Weishaupt KR. The structure of the active component of hematoporphyrin derivative. IN Doiron DR, Gomer CJ, editors. Porphyrin localization and treatment of tumors. New York, NY: Alan R Liss; 1984. 
8. Hasan T. Photosensitizer delivery mediated by macromolecular carrier systems. In: Dougherty TJ, Henderson BW, editors. Photodynamic therapy: basic principals and clinical applications. New York, NY: Marcel Dekker; 1992:187-200.

9. Strong L, Yarmush DM, Yarmush ML. Antibody-targeted photolysis. Photophysical, biochemical, and pharmacokinetic properties of antibacterial conjugates Ann N Y Acad Sci. 1994 Nov 30;745:297-320.

10. Parrish JA, Anderson RR, Urbach F, Pitts D. Optical properties of the skin and eyes. UV-A: biological effects of ultraviolet radiation with emphasis on human responses to longwave ultraviolet. New York, NY: Plenum; 1978.

11. Svaasand LO. Ellingsen R. Optical properties of human brain. Photochem Photobiol. 1983;38(3):293-299.

13. Dougherty TJ, Gomer CJ, Henderson BW, Jori G, Kessel D, Korbelik M et al. Photodynamic therapy. J Natl Cancer Inst. 1998 Jun 17;90(12):889-905.

14. Dougherty TJ. Photosensitizers: therapy and detection of malignant tumors Photochem Photobiol. 1987 Jun;45(6): 879-889.

15. Henderson BW, Dougherty TJ. Photodynamic therapy: basic principles and clinical applications. New York, NY: Marcel Dekker; 1992.

16. Gomer CJ. Preclinical examination of first and second generation photosensitizers used in photodynamic therapy. Photochem Photobiol. 1991 Dec;54(6):1093-107.

17. Boyle RW. Dolphin D. Structure and biodistribution relationships of photodynamic sensitizers. Photochem Photobiol. 1996 Sep;64(3):469-485.

18. Schmidt-Erfurth U, Miller J, Sickenberg M, Bunse A, Laqua H, Gragoudas E et al. Photodynamic therapy of subfoveal choroidal neovascularization: clinical and angiographic examples. Graefes Arch Clin Exp Ophthalmol. 1998 May;236(5):365-374.

19. Miller JW, Schmidt-Erfurth U, Sickenberg M, Pournaras CJ, Laqua H, Barbazetto I, et al.. Photodynamic therapy with verteporfin for choroidal neovascularization caused by age-related macular degeneration: results of a single treatment in a phase 1 and 2 study. Arch Ophthalmol. 1999 Sep;117(9):1161-1173.

20. Schmidt-Erfurth U, Flotte TJ, Gragoudas ES, Schomacker K, Birngruber R, Hasan T. Benzoporphyrin-lipoproteinmediated photodestruction of intraocular tumors. Exp Eye Res. 1996 Jan;62(1):1-10.

21. Schmidt-Erfurth U, Bauman W, Gragoudas E, Flotte TJ, Michaud NA, Birngruber R et al. Photodynamic therapy of experimental choroidal melanoma using lipoprotein-delivered benzoporphyrin. Ophthalmology. 1994 Jan;101(1): 89-99.

22. Schmidt-Erfurth U. Photodynamic therapy. Minimally invasive treatment of choroidal neovascularization. Ophthalmologe. 1998 Oct;95(10):725-731.

23. Oseroff AR, Ohuoha D, Hasan T, Bommer JC, Yarmush ML. Antibody-targeted photolysis: selective photodestruction of human T-cell leukemia cells using monoclonal antibody-chlorin e6 conjugates. Proc Natl Acad Sci U S A. 1986 Nov;83(22):8744-8748.

24. Cincotta L, Szeto D, Lampros E, Hasan T, Cincotta AH. Benzophenothiazine and benzoporphyrin derivative combination phototherapy effectively eradicates large murine sarcomas. Photochem Photobiol. 1996 Feb;63(2):229-237.
25. Cincotta L, Foley JW, Cincotta AH. Novel red absorbing benzo[a]phenoxazinium and benzo[a]phenothiazinium photosensitizers: in vitro evaluation. Photochem Photobiol. 1987 Nov;46(5):751-758.

26. Cincotta L, Foley JW, MacEachern T, Lampros E, Cincotta AH. Novel photodynamic effects of a benzophenothiazine on two different murine sarcomas. Cancer Res. 1994 Mar 1;54(5):1249-1258.

27. Koenig F, McGovern FJ, Larne R, Enquist H, Schomacker KT, Deutsch TF. Diagnosis of bladder carcinoma using protoporphyrin IX fluorescence induced by 5-aminolaevulinic acid. BJU Int. 1999 Jan;83(1):129-135.

28. Baumgartner R, Huber RM, Schulz H, Stepp H, Rick K, Gamarra F, et al. Inhalation of 5-aminolevulinic acid: a new technique for fluorescence detection of early stage lung cancer. J Photochem Photobiol B. 1996 Nov;36(2): 169-174.

29. Leunig A, Rick K, Stepp H, Gutmann R, Alwin G, Baumgartner R, Feyh J. Fluorescence imaging and spectroscopy of 5-aminolevulinic acid induced protoporphyrin IX for the detection of neoplastic lesions in the oral cavity. Am J Surg. 1996 Dec;172(6):674-677.

30. Mayinger B, Reh H, Hochberger J, Hahn EG. Endoscopic photodynamic diagnosis: oral aminolevulinic acid is a marker of GI cancer and dysplastic lesions. Gastrointest Endosc. 1999 Aug;50(2):242-246.

31. Eker C, Montán S, Jaramillo E, Koizumi K, Rubio C, Andersson-Engels S, et al. Clinical spectral characterisation of colonic mucosal lesions using autofluorescence and delta aminolevulinic acid sensitisation. Gut. 1999 Apr;44 (4):511-518.

32. Barnett AA, Gannon M, Brown SB. The use of 5-aminolevulinic acid for photodiagnosis in the endometrium during hysteroscopy. Presented at the Eighth Congress of the European Society for Photobiology. Granada, Spain: European Society for Photobiology; 1999.

33. Roberts WG, Hasan T. Tumor-secreted vascular permeability factor/vascular endothelial growth factor influences photosensitizer uptake. Cancer Res. 1993 Jan 1;53(1):153-157.

34. Nelson JS, Liaw LH, Orenstein A, Roberts WG, Berns MW. Mechanism of tumor destruction following photodynamic therapy with hematoporphyrin derivative, chlorin, and phthalocyanine. J Natl Cancer Inst 1988 Dec 21;80(20): 1599-1605.

35. Reed MW, Wieman TJ, Schuschke DA, Tseng MT, Miller FN. A comparison of the effects of photodynamic therapy on normal and tumor blood vessels in the rat microcirculation. Radiat Res. 1989 Sep;119(3):542-552.

36. Roberts WG, Hasan T. Role of neovasculature and vascular permeability on the tumor retention of photodynamic agents. Cancer Res. 1992 Feb 15;52(4):924-930.

37. Jori G, Beltramini M, Reddi E, Salvato B, Pagnan A, Ziron L, Tomio L, Tsanov T. Evidence for a major role of plasma lipoproteins as hematoporphyrin carriers in vivo. Cancer Lett. 1984 Oct;24(3):291-297.

38. Kesse D. Porphyrin-lipoprotein association as a factor in porphyrin localization. Cancer Lett. 1986 Nov;33(2):183-188.

39. Lange N, Jichlinski $P$, Zellweger M, Forrer M, Marti A, Guillou L, et al. Photodetection of early human bladder cancer based on the fluorescence of 5-aminolaevulinic acid hexylester-induced protoporphyrin IX: A pilot study. Br J Cancer. 1999 Apr;80(1-2):185-193. 
40. Uehlinger P, Zellweger M, Wagnieres G, et al. 5-Aminolevulinic acid and its derivatives: physical chemical properties and protoporphyrin IX formation in cultured cells. J Photochem Photobiol B. 2000 Jan;54(1):72-80.

41. Uehlinger P, Zellweger M, Wagnières G, Juillerat-Jeanneret $\mathrm{L}$, van den Bergh $\mathrm{H}$, Lange $\mathrm{N}$. Tumor-secreted vascular permeability factor/vascular endothelial growth factor influences photosensitizer uptake. Cancer Res 1993;53: 153-157.

42. Colussi VC, Feyes DK, Mulvihill JW, Li YS, Kenney ME, Elmets CA, Oleinick NL, Mukhtar H. Phthalocyanine 4 (Pc 4) photodynamic therapy of human OVCAR-3 tumor xenografts. Photochem Photobiol. 1999 Feb;69(2):236-241.

43. Wood SR, Holroyd JA, Brown SB. The subcellular localization of $\mathrm{Zn}$ (II) phthalocyanines and their redistribution on exposure to light. Photochem Photobiol. 1997 Mar;65(3): 397-402.

44. Gupta S, Ahmad N, Mukhtar H. Involvement of nitric oxide during phthalocyanine (Pc4) photodynamic therapy-mediated apoptosis. Cancer Res. 1998 May 1;58(9):17851788.

45. Kim HR, Luo Y, Li G, Kessel D. Enhanced apoptotic response to photodynamic therapy after bcl-2 transfection. Cancer Res. 1999 Jul 15;59(14):3429-3432.

46. Pogue BW, Redmond RW, Trivedi N, Hasan T. Photophysical properties of tin ethyl etiopurpurin I (SnET2) and tin octaethylbenzochlorin (SnOEBC) in solution and bound to albumin. Photochem Photobiol. 1998 Dec;68(6):809815.

47. Ahmad N, Mukhtar H. Mechanism of photodynamic therapy-induced cell death. Methods Enzymol. 2000;319:342358.

48. Kim HR, Luo Y, Li G, Kessel D. Enhanced apoptotic response to photodynamic therapy after bcl-2 transfection. Cancer Res. 1999 Jul 15;59(14):3429-3432.

49. Agarwal ML, Larkin HE, Zaidi SI, Mukhtar H, Oleinick NL. Phospholipase activation triggers apoptosis in photosensitized mouse lymphoma cells. Cancer Res. 1993 Dec 15;53 (24):5897-5902.

50. Agostinis P, Assefa Z, Vantieghem A, Vandenheede JR, Merlevede W, De Witte P. Apoptotic and anti-apoptotic signaling pathways induced by photodynamic therapy with hypericin. Adv Enzyme Regul. 2000;40:157-182.

51. Xue L, He J, Oleinick NL. Promotion of photodynamic therapy-induced apoptosis by stress kinases. Cell Death Differ. 1999 Sep;6(9):855-864.

52. Xue LY, Qiu Y, He J, Kung HJ, Oleinick NL. Etk/Bmx, a $\mathrm{PH}$-domain containing tyrosine kinase, protects prostate cancer cells from apoptosis induced by photodynamic therapy or thapsigargin. Oncogene. 1999 Jun 3;18(22):33913398.

53. Xue LY, He J, Oleinick NL. Rapid tyrosine phosphorylation of HS1 in the response of mouse lymphoma L5178Y$\mathrm{R}$ cells to photodynamic treatment sensitized by the phthalocyanine Pc 4. Photochem Photobiol. 1997 Jul;66(1): 105-113.

54. Gomer CJ, Ryter SW, Ferrario A, Rucker N, Wong S, Fisher AM. Photodynamic therapy-mediated oxidative stress can induce expression of heat shock proteins. Cancer Res. 1996 May 15;56(10):2355-2360.

55. Gomer CJ, Ferrario A, Rucker N. Glucose regulated protein induction and cellular resistance to oxidative stress mediated by porphyrin photosensitization. Cancer Res. 1991 Dec 15;51(24):6574-6579.
56. Morgan J, Whitaker JE, Oseroff AR. GRP78 induction by calcium ionophore potentiates photodynamic therapy using the mitochondrial targeting dye victoria blue BO. Photochem Photobiol. 1998;67(1):155-164.

57. Xue LY, Agarwal ML, Varnes ME. Elevation of GRP-78 and loss of HSP-70 following photodynamic treatment of V79 cells: sensitization by nigericin. Photochem Photobiol. 1995; 62(1):135-143.

58. Runnels JM, Chen B, Ortel B. BPD-MA-mediated photosensitization in vitro and in vivo: cellular adhesion and B1 integrin expression in ovarian cancer cells. Br J Cancer. 1999;80(7):946-953.

59. Evans S, Matthews W, Perry R. Effect of photodynamic therapy on tumor necrosis factor production by murine macrophages. J Natl Cancer Inst. 1990;82(1):34-39.

60. Hunt DW, Chan AH. Influence of photodynamic therapy on immunological aspects of diseasean update. Expert Opin Investig Drugs. 2000;9(4):807-817.

61. Pogue BW, Ortel B, Redmond RW. A photobiological and photophysical-based study of phototoxicity of two chlorins. Cancer Res. 2001;(2):717-724.

62. Steele JK, Liu D, Stammers AT. Suppressor deletion therapy: selective elimination of $\mathrm{T}$ suppressor cells in vivo using a hematoporphyrin conjugated monoclonal antibody permits animals to reject syngeneic tumor cells. Cancer Immunol Immunother. 1988;26(2):125-31.

63. Gomer CJ, Ferrario A, Murphree AL. The effect of localized porphyrin photodynamic therapy on the induction of tumour metastasis. Br J Cancer. 1987;56(1):27-32.

64. Nseyo UO, Whalen RK, Duncan MR. Urinary cytokines following photodynamic therapy for bladder cancer. A preliminary report. Urology. 1990;36(2):167-171.

65. Gomer CJ, Ferrario A, Hayashi N, Rucker N, Szirth BC, Murphree AL.. Molecular, cellular, and tissue responses following photodynamic therapy. Lasers Surg Med. 1988; 8(5):450-463.

66. Grossweiner LI. Optical dosimetry in photodynamic therapy. Lasers Surg Med. 1986;6(5):462-466.

67. Camps JL Jr, Powers SK, Beckman WC Jr, Brown JT, Weissman RM. Photodynamic therapy of prostate cancer: an in vitro study. J Urol. 1985;134(6):1222-1226.

68. Koudinova NV, Pinthus JH, Brandis A. Photodynamic therapy with Pd-Bacteriopheophorbide (TOOKAD). Successful in vivo treatment of human prostatic small cell carcinoma xenografts. Int J Cancer. 2003;104(6):782-789.

69. Selman SH, Keck RW, Hampton JA. Transperineal photodynamic ablation o the canine prostate. J Urol. 1996; 156(1):258-260.

70. Selman SH, Albrecht D, Keck RW, Brennan P, Kondo S. Studies of tin ethyl etiopurpurin photodynamic therapy of the canine prostate. J Urol. 2001;165(5)1795-1801.

71. Hsi RA, Kapatkin A, Strandberg J et al. Photodynamic therapy in the canine prostate using motexafin lutetium. Clin Cancer es. 2001;7(3):651-660.

72. Lee LK, Whitehurst C, Chen Q, Pantelides ML, Hetzel FW, Moore JV. Interstitial photodynamic therapy in the canine prostate. Br J Urol. 1997;80(6):898-902.

73. Chang SC, Buonaccorsi GA, MacRobert AJ, Bown SG. Interstitial photodynamic therapy in the canine prostate with disulfonated aluminum phthalocyanine and 5-aminolevulinic acid-induced protoporphyrin IX. Prostate 1997; 32(2):89-98. 
74. Huang Z, Chen Q, Trncic N. Effects of Pd-bacteriopheophorbide (TOOKAD) -mediated photodynamic therapy on canine prostate pretreated with ionizing radiation. Radiat Res. 2004;161(6):723-731.

75. Chen Q, Huang Z, Luck D, Beckers J, Brun PH, Wilson BC. Preclinical studies in normal canine prostate of a novel palladium-bacteriopheophorbide (WST09) photosensitizer for photodynamic therapy of prostate cancers. Photochem Photobiol. 2002;76/4):438-445.

76. Windahl T, Andersson SO, Lofgren L. Photodynamic the rapy of localised prostatic cancer. Lancet. 1990;336 (8723): 1139.

77. Nathan TR, Whitelaw DE, Chang SC, Lees WR, Ripley PM, Payne $\mathrm{H}$ et al. Photodynamic therapy for prostate cancer recurrence after radiotherapy: a phase I study. J Urol. 2002; 168(4 pt1):1427-1432.

78. Moore C, Hoh I, Bown S, Emberton M; Does Photodynamic therapy have the necessary attrbutes to become a future treatment for organ confines prostate cancer? $\mathrm{Br}$ Jour Urol. 2005; 96(6):754-758

79. Kubler AC, Stenzel W, Ruhling M, Meul B, Fischer JH. Experimental evaluation of possible side effects of intraoperative photodynamic therapy on rabbit blood vessels and nerves. Lasers Surg Med. 2003;33(4):247-255.

80. Patel HR, Mirsadraee S, Emberton M. The patient's dilemma: prostate cancer treatment choices. J Urol. 2003;169(3): 828-833.

Correspondencia autor: Dr. M. Ramírez Backhaus Servicio de Urología. Hospital Universitario La Fe Avda. Campanar, 21 - 46009 Valencia Tel.: 963862700

E-mail autor: ramirezbackhaus@yahoo.es Información artículo: Revisión 